\title{
Ombitasvir, Paritaprevir, Ritonavir, and Dasabuvir Mini-Tabs Plus Ribavirin for Children Aged 3-11 Years with Hepatitis C Genotype 1a
}

Philip Rosenthal - Michael R. Narkewicz - Betty B. Yao •

Christopher D. Jolley · Steven J. Lobritto · Jessica Wen •

Jean P. Molleston · Evelyn K. Hsu • Maureen M. Jonas •

Jiuhong Zha $\cdot$ Li Liu $\cdot$ Daniel H. Leung

Received: March 17, 2020 / Published online: May 25, 2020

(C) The Author(s) 2020

\section{ABSTRACT}

Introduction: To assess the safety, efficacy, and pharmacokinetics of mini-tablet formulations of ombitasvir (OBV), paritaprevir (PTV), ritonavir, and dasabuvir (DSV) with or without ribavirin for 12 weeks in children infected with chronic hepatitis $\mathrm{C}$ virus (HCV) genotype (GT) 1.

Electronic supplementary material The online version of this article (https://doi.org/10.1007/s12325020-01389-9) contains supplementary material, which is available to authorized users.

Digital Features To view digital features for this article go to https://doi.org/10.6084/m9.figshare.12293216

P. Rosenthal $(\bowtie)$

Department of Pediatrics, University of California,

San Francisco, CA, USA

e-mail: PROSENTH@ucsf.edu

\section{R. Narkewicz}

Digestive Health Institute, Children's Hospital Colorado and Section of Pediatric Gastroenterology,

Hepatology, and Nutrition, Department of

Pediatrics, University of Colorado School of

Medicine, Aurora, CO, USA

B. B. Yao $\cdot$ J. Zha $\cdot$ L. Liu

AbbVie Inc., North Chicago, IL, USA

C. D. Jolley

Department of Pediatrics, Gastroenterology,

Hepatology, and Nutrition, University of Florida,

Gainesville, FL, USA
Methods: This is an ongoing, open-label, Phase $2 / 3$ study in children $3-11$ years old infected with HCV GT1 who were HCV treatment-naïve and non-cirrhotic. Pediatric mini-tablet formulations of OBV, PTV, ritonavir, and DSV plus ribavirin oral solution were administered for 12 weeks based on body weight. Endpoints included SVR12, adverse events (AEs), and pharmacokinetic parameters.

Results: Overall, 26 children received OBV, PTV, ritonavir, and DSV plus ribavirin; 14 were $3-8$ years old and 12 were $9-11$ years old; $35 \%$ were male; and all had chronic HCV GT1a infection. The SVR12 rate was 96\% (25/26; 95\% CI 81.1-99.3), with 1 child failing to achieve SVR12 due to non-adherence and treatment discontinuation. Treatment-emergent AEs of

\section{S. J. Lobritto}

NewYork Presbyterian, Morgan Stanley Children's Hospital and Department of Pediatrics, Columbia University Irving Medical Center, New York, NY, USA

J. Wen

Division of Gastroenterology, Hepatology and Nutrition, The Children's Hospital of Philadelphia and Department of Pediatrics, University of Pennsylvania, Philadelphia, PA, USA

\section{J. P. Molleston}

Department of Pediatrics, Indiana University School of Medicine, Riley Hospital for Children, Indianapolis, IN, USA 
Grade $\geq 3$ occurred in 3 children; 2 events in 1 child were considered serious; and none were considered treatment-related. No AEs led to discontinuation of study treatment. The most common AEs were headache (27\%), fatigue (23\%), pyrexia (19\%), and vomiting (19\%). Pharmacokinetic results showed mini-tablet formulations of OBV, PTV, DSV, and ritonavir drug exposures were comparable to the adult formulation.

Conclusion: The mini-tablet combination of OBV, PTV, ritonavir, and DSV plus ribavirin to treat HCV GT1a infection for 12 weeks was highly effective and suitable in children 3-11 years of age.

Trial Registration: ClinicalTrials.gov identifier, NCT02486406.

Keywords: Efficacy; Hepatology; Interferonfree DAA regimens; Pediatrics; Pharmacokinetics; Safety

\section{Key Summary Points}

Why carry out this study?

The efficacy and safety of regimens approved for treating chronic hepatitis $\mathrm{C}$ virus (HCV) infection in adults remain to be studied in children (3-11 years) with $\mathrm{HCV}$ infection.

Guidelines recommend treatment with available direct-acting antiviral (DAA) regimens where available or deferring treatment until alternative interferon-free regimens are available for this age group.

\section{E. K. Hsu}

Seattle Children's Hospital and Department of Pediatrics, University of Washington School of Medicine, Seattle, WA, USA

M. M. Jonas

Division of Gastroenterology, Hepatology, and Nutrition, Boston Children's Hospital and

Department of Pediatrics, Harvard Medical School, Boston, MA, USA

\section{H. Leung}

Baylor College of Medicine, Gastroenterology, Hepatology, and Nutrition, Texas Children's Liver Center, Houston, TX, USA

\section{What was learned from the study?}

We report the safety, efficacy, and pharmacokinetics of a pediatric 'minitablet' formulation of the drug combination ombitasvir, paritaprevir, ritonavir, and dasabuvir with ribavirin in children 3-11 years of age with HCV GT1a infection.

The safety profile was similar to that seen in adults and adolescents treated with the adult formulation, supporting the use of DAAs in children.

\section{INTRODUCTION}

The clinical course of hepatitis C virus (HCV) infection in children differs from that acquired during later life [1]. Perinatal transmission is the primary route of infection, with approximately $5 \%$ of chronically infected mothers transmitting HCV to their children $[1,2]$. Approximately $20 \%$ of children will spontaneously clear the virus, usually before their fourth year of life [3]. The remaining 80\% will develop chronic HCV infection that persists into adulthood [3], and, although usually benign [4], complications associated with HCV-related liver disease are still a risk for pediatric patients [1]. Although an estimated 11 million children $(<15$ years) are $\mathrm{HCV}$ antibody-positive worldwide (6 million of whom are viremic) [5], this may be grossly underestimated due to the asymptomatic disease course and inadequate screening programs $[1,6]$.

All-oral, interferon (IFN)-free, direct-acting antiviral (DAA) drugs are the standard of care for the treatment of adults and adolescents aged 12-17 years infected with any HCV genotype (GT). These drugs are consistently associated with high rates of sustained virologic response (SVR) and are generally well tolerated $[3,7,8]$. Due to the large variance in age and weight in a pediatric population $<12$ years old, pediatric formulations of medications may be necessary to meet appropriate efficacy and safety parameters [9]. 
Recently, 2 IFN-free DAA regimens, sofosbuvir and ledipasvir/sofosbuvir, have been approved for treating children aged $<12$ years $[10,11]$. Previously, the only available treatment option was pegylated IFN (pegIFN) combined with ribavirin (RBV) [3]. Discontinuation rates are much lower with IFN-free DAAs than pegIFNbased regimens, and virologic response, as well as safety and tolerability, are greatly improved [3]. As a result, current guidelines recommend treatment with available DAA regimens for children aged 3-11 years with chronic HCV or deferment of treatment until IFN-free regimens are available $[3,7,8]$.

The combination DAA treatment of ombitasvir (OBV) and paritaprevir (PTV) boosted with the pharmacokinetic enhancer, ritonavir $(\mathrm{r})$, and co-administered with or without dasabuvir (DSV) and with or without RBV $(\mathrm{OBV} / \mathrm{PTV} / \mathrm{r} \pm \mathrm{DSV} \pm \mathrm{RBV})$ is approved for treating adults infected with HCV GT1 or GT4, without cirrhosis or with compensated cirrhosis [12-14]. OBV/PTV/r $\pm \mathrm{DSV} \pm \mathrm{RBV}$ regimens are associated with high rates of SVR $(>90 \%)$ at post-treatment Week 12 (SVR12) when administered for up to 24 weeks across the broad range of approved patient populations $[11,12]$.

The ZIRCON study (NCT02486406), designed to evaluate the safety, efficacy, and pharmacokinetics of OBV, PTV, and ritonavir with or without DSV and with or without RBV in children and adolescents infected with HCV GT1 or GT4, showed them to be well tolerated and resulted in SVR12 rates of 100\% in $38 \mathrm{HCV}$ infected adolescents (12-17 years) [15]. Here, we report on the safety and efficacy in children aged 3-11 years infected with HCV GT1 who received pediatric formulations of the study drugs. The pharmacokinetic results across all age groups (children and adolescents) are also reported here.

\section{METHODS}

\section{Study Design and Participants}

ZIRCON (NCT02486406) is an ongoing, openlabel, multinational study. It was designed to evaluate the pharmacokinetics, safety, and efficacy of individually formulated OBV, PTV, ritonavir, DSV mini-tablets with or without RBV oral solution (pediatric formulation) in children aged 3-11 years, who were infected with HCV GT1, treatment-naïve, and without cirrhosis. The study was designed to allow dose adjustments of OBV, PTV, ritonavir, and DSV to achieve optimal therapeutic exposure to the mini-tablet formulations.

The study enrolled chronic HCV-infected children aged 3-11 years who were treatment-naïve, weighing $\geq 15 \mathrm{~kg}$, with HCV GT1 [positive anti-HCV antibody test and HCV ribonucleic acid (RNA) concentration $\geq 1000 \mathrm{IU} / \mathrm{mL}$ at screening] without cirrhosis in the United States between July 2017 and November 2017. At least 12 children aged 9-11 years ( $\geq 4$ with a body weight $15-44 \mathrm{~kg}$ ), and at least 12 children aged $3-8$ years $(\geq 2$ children from each of the age groups 3-5 years and 6-8 years) were planned for enrollment. Absence of cirrhosis was based on liver biopsy (Metavir score $\leq 3$, Ishak score $\leq 4$ ) or a FibroScan $^{\mathrm{TM}}$ (Echosens, Paris, France) result of $<14.6 \mathrm{kPa}$ within the 24 months before screening. If neither biopsy nor FibroScan results were available, FibroTest ${ }^{\mathrm{TM}}$ (BioPredictive, Paris, France) was performed at screening and a score of $<0.75$ confirmed absence of cirrhosis.

Key exclusion criteria included coinfection with hepatitis B virus (HBV) or human immunodeficiency virus (HIV); current or past clinical evidence of Child-Pugh B or C classification (Child-Pugh score $\geq 7$ ) or clinical history of liver decompensation; hepatocellular carcinoma; previous or current use of any investigational or commercially available antiHCV drug; history of solid organ transplantation; or any of the following abnormal laboratory results: albumin $<2.8 \mathrm{~g} / \mathrm{dL}$, hemoglobin $<10 \mathrm{~g} / \mathrm{dL}$, platelets $<25,000$ cells/ $\mathrm{mm}^{3}$, or total bilirubin $>3.0 \mathrm{mg} / \mathrm{dL}$. Full eligibility criteria are provided in S1.

Eligible children were allocated to receive 12 weeks of treatment with OBV, PTV, ritonavir, and DSV mini-tablet formulations, administered in a dosing vehicle of soft food with or without RBV solution, depending on the HCV 
Table 1 Weight-based dosing regimen

\begin{tabular}{llllll}
\hline Body weight $(\mathbf{k g})$ & OBV QD (mg) & PTV QD (mg) & Ritonavir QD (mg) & DSV BID $(\mathbf{m g})$ & $\begin{array}{l}\text { RBV } \mathbf{4 0 ~} \mathbf{m g} / \mathbf{m L} \\
\text { oral solution }^{\mathbf{a}}\end{array}$ \\
\hline $15-29$ & 10 & 50 & 35 & 100 & GT1a only \\
$30-44$ & 15 & 100 & 70 & 150 & GT1a only \\
$\geq 45$ & 25 & 150 & 100 & 250 & GT1a only \\
\hline
\end{tabular}

$B I D$ twice daily, $D S V$ dasabuvir, $G T$ genotype, $O B V$ ombitasvir, $P T V$ paritaprevir, $Q D$ once daily, $R B V$ ribavirin

${ }^{a}$ Weight-based RBV administered via dosing syringe according to the local label in each country

GT1 subtype. All HCV GT1a-infected patients received weight-based RBV; dosing was in accordance to the US local label. Children aged 9-11 years were enrolled and treated first. Following acceptable efficacy, safety, and pharmacokinetic data in at least six children, children aged 3-8 years were enrolled and treated. All children received treatment based on body weight (Table 1).

All participants, irrespective of whether they prematurely discontinued study treatment, were followed up for an initial 24-week posttreatment period. The durability of response, emergence, and persistence of drug resistance, and the impact of study treatment on participants' growth and development were assessed until post-treatment Week 144 in those who completed the post-treatment Week-24 visit.

Children with virologic failure during the treatment period were discontinued from study treatment per protocol. Virologic failure was defined as two consecutive HCV RNA measurements $>1 \log 10 \mathrm{IU} / \mathrm{mL}$ above nadir at any time point or two consecutive HCV RNA measurements $\geq 100 \mathrm{IU} / \mathrm{mL}$ at any point after achieving HCV RNA less than the lower limit of quantification (LLOQ).

The study protocol and amendments were approved by the independent ethics committee or institutional review board for each study site. IRB/IEC approval was given to the study protocol by: Columbia University Medical Center; Boston Children's Hospital; the Committees for Protection of Human Subjects at the Children's Hospital of Philadelphia; the Western Institutional Review Board (WIRB) at the University of Florida; WIRB at Seattle Children's Hospital;
WIRB at Baylor College of Medicine; WIRB at the Children's Hospital Colorado; the Indiana University Office of Research Administration, Human Subjects Office; the Human Research Protection Program IRB at the University of California, San Francisco. The study was designed and conducted in accordance with the International Conference on Harmonization Good Clinical Practice guidelines and the ethical principles of the Declaration of Helsinki. Parents or legal guardians provided written informed consent, and participants gave assent, as appropriate for their age and country. All authors had access to study data and reviewed and approved the final manuscript.

\section{Efficacy Assessments}

The primary efficacy endpoint was the percentage of children who achieved SVR12, defined as an HCV RNA concentration less than the LLOQ at post-treatment Week 12. Secondary efficacy endpoints were the percentages of children who achieved SVR12 by age group (9-11 years and $3-8$ years) and by weight range and the percentages of children with normalization of alanine aminotransferase (ALT) concentrations during treatment [ALT $\leq$ central laboratory's upper limit of normal (ULN); $34 \mathrm{U} /$ $\mathrm{L}$ for females and $43 \mathrm{U} / \mathrm{L}$ for males] by age and weight group at the final treatment visit for children with ALT above ULN at baseline.

Blood samples were collected to determine HCV RNA concentrations at screening; on treatment Day 1; during treatment Weeks 2, 4, 8, and 12; and post-treatment Weeks 4, 12, 24, $36,48,96$, and 144. HCV RNA concentrations 
Table 2 Baseline demographics and clinical characteristics (ITT population)

\begin{tabular}{|c|c|c|c|c|}
\hline & \multicolumn{2}{|c|}{$9-11$ years of age } & \multirow{2}{*}{$\begin{array}{l}3-8 \text { years of age } \\
15-29 \mathrm{~kg}\end{array}$} & \multirow[t]{2}{*}{ Total } \\
\hline & $15-44 \mathrm{~kg}^{\mathrm{a}}$ & $\geq 45 \mathrm{~kg}$ & & \\
\hline$n$ & 10 & 2 & 14 & 26 \\
\hline Male & $5(50)$ & $1(50)$ & $3(21)$ & $9(35)$ \\
\hline Age, years, median [range] & $9.5[9.0-11.0]$ & $10.5[10.0-11.0]$ & $4.5[3.0-8.0]$ & $7.5[3.0-11.0]$ \\
\hline \multicolumn{5}{|l|}{ Race } \\
\hline White & $8(80)$ & $2(100)$ & $10(71)$ & $20(77)$ \\
\hline Black or African American & 0 & 0 & $4(29)$ & $4(15)$ \\
\hline Asian & $1(10)$ & 0 & 0 & $1(4)$ \\
\hline Multi-racial & $1(10)$ & 0 & 0 & $1(4)$ \\
\hline \multicolumn{5}{|l|}{ Ethnicity } \\
\hline Hispanic or Latino & $1(10)$ & 0 & $3(21)$ & $4(15)$ \\
\hline BMI, $\mathrm{kg} / \mathrm{m}^{2}$ & $17.1 \pm 1.8$ & $21.0 \pm 5.8$ & $16.5 \pm 1.3$ & $17.1 \pm 2.2$ \\
\hline \multicolumn{5}{|l|}{ HCV genotype/subtype } \\
\hline GT1a & $10(100)$ & $2(100)$ & $14(100)$ & $26(100)$ \\
\hline $\mathrm{HCV}$ RNA, $\log 10 \mathrm{IU} / \mathrm{mL}$ & $6.1 \pm 0.8$ & $5.6 \pm 0.4$ & $5.6 \pm 0.5$ & $5.8 \pm 0.7$ \\
\hline$\geq 800,000 \mathrm{IU} / \mathrm{mL}$ & $7(70)$ & 0 & $4(29)$ & $11(42)$ \\
\hline IL28B genotype, non-CC ${ }^{\mathrm{b}}$ & $5(50)$ & $2(100)$ & $11(79)$ & $18(69)$ \\
\hline \multicolumn{5}{|l|}{ Fibrosis stage ${ }^{c}$} \\
\hline F0-F1 & $8(80)$ & $1(50)$ & $13(93)$ & $22(85)$ \\
\hline $\mathrm{F} 2$ & $2(20)$ & $1(50)$ & 0 & $3(12)$ \\
\hline F3 & 0 & 0 & $1(7)$ & $1(4)$ \\
\hline
\end{tabular}

Data are $n(\%)$ or mean \pm SD unless stated otherwise

$B M I$ body mass index, IL28B interleukin 28B, ITT intention-to-treat, $S D$ standard deviation

a There was 1 patient in the $15-29 \mathrm{~kg}$ group. Baseline characteristics were combined to protect patient identity

b Single nucleotide polymorphism $=$ RS12979860

${ }^{c} F 0-F 1$ liver biopsy score 0 or 1 , Ishak score $1-2$, FibroScan ${ }^{\mathrm{TM}}<8.8 \mathrm{kPa}$ or FibroTest ${ }^{\mathrm{TM}}<0.49 ;$; 2 liver biopsy score 2, Ishak score 3, FibroScan 8.8 to $<9.6 \mathrm{kPa}$, or FibroTest 0.49 to $<0.59$; F3 liver biopsy score 3, Ishak score 4, FibroScan 9.6 to $<14.6 \mathrm{kPa}$, or FibroTest 0.59 to $<0.75$

were determined at a central laboratory (Covance $^{\circledR}$ Central Laboratory Services) using the COBAS $^{\circledR}$ AmpliPrep/COBAS $^{\circledR}$ TaqMan $^{\circledR}$ HCV Test (Roche, Basel, Switzerland), v2.0 (LLOQ and lower limit of detection: $15 \mathrm{IU} / \mathrm{mL}$ ).

\section{Safety Assessments}

The following safety evaluations were undertaken at each visit during the 12-week treatment period: assessment of adverse events (AEs) and vital signs (up to post-treatment Week 44), 
and clinical laboratory findings (up to posttreatment Week 4), along with a symptom-directed physical examination when indicated. All AEs that occurred between the first dose of study treatment and 30 days after the last dose were recorded and coded according to the Medical Dictionary for Regulatory Activities (MedDRA MSSO, McLean, VA, USA) System Organ Class and preferred term (v.21.0). The relatedness of AEs to DAA or RBV treatment and the severity of AEs [according to the National Cancer Institute Common Terminology Criteria for Adverse Events (CTCAE) criteria] were assessed by the investigator.

The following growth and development endpoints were measured through post-treatment Week 24: growth rate at each post-baseline visit (defined as change in height divided by change in age from the previous visit), height $z$ score, waist circumference, and Tanner staging.

\section{Pharmacokinetic Assessments}

Blood samples were collected for intensive pharmacokinetic analysis as described in S2.

\section{Acceptability of Pediatric Mini-Tablet Formulations}

The parents or guardians of the participants were asked to complete acceptability questionnaires at Week 2 and Week 12, to provide feedback on the acceptability of the mini-tablet formulations. They were asked about their overall impression of the formulations and their administration via a dosing vehicle, including time taken to administer, volume of dosing vehicle, palatability, and convenience.

\section{Statistical Analysis}

All data were analyzed using the SAS ${ }^{\circledR}$ software package (SAS Institute, Cary, NC, USA). For

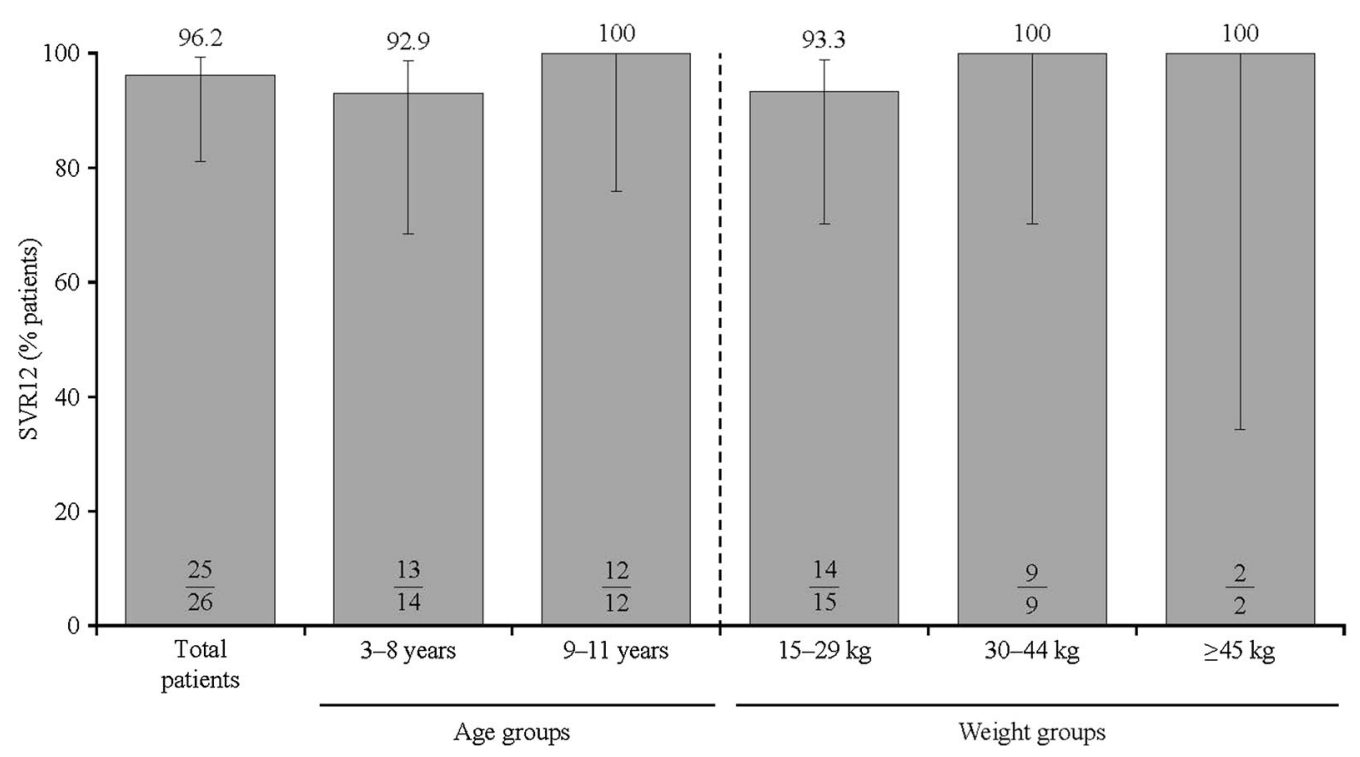

Fig. 1 SVR12 rates by age and weight group following treatment with OBV, PTV, ritonavir, and DSV mini-tablet formulations plus RBV solution for 12 weeks (ITT population). Error bars represent $95 \%$ confidence intervals, which were calculated using the Wilson's score method. Backward imputation, where applicable, was used to impute missing data. After applying backward imputation, if there was still no value in the window but there was an HCV RNA value from a local laboratory present, then it was imputed into the SVR window; otherwise, patients with missing data were counted as virologic failures. $D S V$ dasabuvir, $H C V$ hepatitis $\mathrm{C}$ virus, ITT intention-to-treat, $O B V$ ombitasvir, $P T V$ paritaprevir, $R B V$ ribavirin, $R N A$ ribonucleic acid, SVRI2 sustained virologic response (>90\%) at post-treatment Week 12 
Table 3 Summary of AEs and laboratory abnormalities (safety population)

\begin{tabular}{|c|c|}
\hline $\mathbf{A E}$ & $\begin{array}{l}\text { Patients, } \\
n=26\end{array}$ \\
\hline Any $\mathrm{AE}$ & $21(81)$ \\
\hline Any AE possibly related to $\mathrm{DAAs} \mathrm{s}^{\mathrm{a}}$ & $10(38)$ \\
\hline Any $A E$ possibly related to $\mathrm{RBV}^{\mathrm{a}}$ & $10(38)$ \\
\hline $\begin{array}{l}\text { Any AE leading to discontinuation of study } \\
\text { drug }\end{array}$ & 0 \\
\hline Any serious $\mathrm{AE}$ & $1(4)$ \\
\hline \multicolumn{2}{|l|}{ AEs in $\geq 10 \%$ of all children } \\
\hline Headache & $7(27)$ \\
\hline Fatigue & $6(23)$ \\
\hline Pyrexia & $5(19)$ \\
\hline Vomiting & $5(19)$ \\
\hline Cough & $4(15)$ \\
\hline Nausea & $4(15)$ \\
\hline Upper respiratory tract infection & $4(15)$ \\
\hline Diarrhea & $3(12)$ \\
\hline Nasopharyngitis & $3(12)$ \\
\hline Laboratory abnormalities & $\begin{array}{l}\text { Patients, } \\
n=26\end{array}$ \\
\hline Hemoglobin $<11.5 \mathrm{~g} / \mathrm{dL}$ & $3 / 25(12)$ \\
\hline Alkaline phosphatase $>1.5 \times \mathrm{ULN}$ & 0 \\
\hline Total bilirubin $\geq 2 \times$ ULN & $3 / 25(12)$ \\
\hline $\begin{array}{l}\text { Alanine aminotransferase post- } \\
\text { nadir }>5 \times \mathrm{ULN}\end{array}$ & 0 \\
\hline
\end{tabular}

$A E$ adverse event, $D A A s$ direct-acting antivirals, $R B V$ ribavirin, $U L N$ upper limit of normal

Data are $n(\%)$

${ }^{a}$ As assessed by investigator

analysis of the primary and secondary efficacy endpoints, the percentages of children who achieved SVR12 were calculated with two-sided 95\% confidence intervals (CIs) in the intentionto-treat (ITT) population, which comprised all patients who had received at least one dose of study treatment. The 95\% CIs were calculated using the normal approximation to the binomial distribution if the rate was not $0 \%$ or 100\%; otherwise, the Wilson's score method was used. Backward imputation was used to impute missing data for SVR endpoints only. If there was no value in the window, but there was an HCV RNA value after the window, it was imputed into the SVR window; otherwise, participants were considered failures.

Safety was analyzed in all children who received at least one dose of study treatment (safety population) and summarized descriptively.

A planned sample size of 12 participants in each of the age groups 3-8 years and 9-11 years was considered adequate to characterize the pharmacokinetics of the mini-tablet formulations.

\section{RESULTS}

\section{Patients}

Between July and November 2017, 26 children (aged 3-11 years) with chronic HCV GT1 infection, who were HCV treatment-naïve and noncirrhotic, were enrolled into the study in the United States. Demographics and clinical characteristics at baseline are presented in Table 2 . In total, 12 children were aged 9-11 years; 1 weighed $15-29 \mathrm{~kg}$, 9 weighed $30-44 \mathrm{~kg}$, and 2 weighed $\geq 45 \mathrm{~kg}$. Fourteen children were aged $3-8$ years, all of whom weighed $15-29 \mathrm{~kg}$. All children were infected with HCV GT1a; therefore, all received OBV, PTV, ritonavir, and DSV mini-tablets plus RBV solution for 12 weeks. Baseline fibrosis stage by historical liver biopsy within 24 months before screening or by FibroTest was F0-F1 in 22 children, F2 in 3 children (all aged 9-11 years), and F3 in 1 child (aged 3-8 years).

Treatment adherence was $\geq 90 \%$ for all mini-tablets and the RBV solution. The median duration of treatment was 85 days (range 9-92 days). One 3-year-old child was discontinued from study treatment after 9 days due to nonadherence (difficulty taking study drugs), but remained in the study for post-treatment follow-up. The administration of study treatment 
was interrupted for 2 days in one 4-year-old child owing to a non-treatment-related $\mathrm{AE}$ (Grade 2 viral gastroenteritis); the patient completed the full 12-week treatment course after the study drugs were resumed. There were no dose modifications to the DAA treatment; the RBV dose was modified in one 10-year-old child owing to decreased hemoglobin level and hyperbilirubinemia. For all patients with documented hemoglobin values at baseline and at the final treatment visit, the mean change in hemoglobin from baseline was $-0.32 \mathrm{~g} / \mathrm{dL} \pm$ 0.73 . The mean change in hemoglobin from baseline at the final post-treatment visit was $-0.05 \mathrm{~g} / \mathrm{dL} \pm 0.73$. All children completed the post-treatment Week 12 visit and were entered into long-term follow-up.

\section{Efficacy}

All but one child treated with OBV, PTV, ritonavir, and DSV mini-tablets plus RBV solution achieved the primary endpoint; the SVR12 rate was $96.2 \%(25 / 26$; 95\% CI 81.1-99.3; ITT analysis). SVR12 rates by age and weight groups are shown in Fig. 1 . The single child who failed to achieve SVR12 was aged 3 years, weighed $18 \mathrm{~kg}$, received study drugs for only 9 days, and had non-virologic failure. In the modified ITT (mITT) analysis, this patient was excluded and the mITT SVR12 rate was $100 \%(25 / 25)$. No virologic failures occurred before or after posttreatment Week 12. Of the 15 children who completed treatment and had elevated ALT concentrations at baseline, 14 [93.3\%; 100\% (6/ 6) aged 9-11 years; $88.9 \%$ (8/9) aged 3-8 years] had ALT normalization during treatment (ALT $\leq \mathrm{ULN}$ at final treatment visit).

\section{Safety}

Overall, 21 children $(81 \% ; 21 / 26)$ experienced AEs during the study (Table 3 ). The most common AEs were headache, fatigue, pyrexia, vomiting, cough, nausea, upper respiratory tract infection, diarrhea, and nasopharyngitis. No AEs led to discontinuation of study treatment, and no deaths occurred during the treatment period. Three children aged 9-11 years had AEs that were
CTCAE Grade $\geq 3$ in severity (MedDRA preferred terms: leukopenia and neutropenia; hyperbilirubinemia; depression), none of which were considered possibly related to DAAs as deemed by the study investigator. Serious AEs of leukopenia (lymphocytes $0.76 \times 10^{3} / \mu \mathrm{L}$ ) and neutropenia $\left(0.35 \times 10^{3} / \mu \mathrm{L}\right)$ occurred on Day 31 in a 9-year-old boy and were assessed to be related to respiratory illness. No AEs that were CTCAE Grade $\geq 3$ occurred in children aged 3-8 years.

Clinically significant post-baseline laboratory abnormalities were infrequent (Table 3). There were no reports of patients with ALT of CTCAE Grade $\geq 3$ (S3). At the final treatment visit, the mean change from baseline for ALT values was $-30.2 \mathrm{U} / \mathrm{L} \pm 22.59$. The mean change from baseline at the final post-treatment visit was $-29.9 \mathrm{U} / \mathrm{L} \pm 22.06$. Vital sign measurements and growth and development outcomes were not affected by study treatment (data not shown).

\section{Pharmacokinetics}

The present study used three different pediatric dose levels based on body weight (Table 1). The doses were selected, based on modeling, to achieve therapeutic exposures that have been demonstrated to be safe and efficacious in adult patients. The actual drug exposures achieved were generally comparable across the three weight groups with overlapping ranges (S4). The individual maximum plasma concentrations $\left(C_{\max }\right)$ and area under the plasma concentration-time curve (AUC) values in the present study were also compared with individual values from historical data from three studies among adult $\mathrm{HCV}$-infected patients who had intensive pharmacokinetic assessment $(n=10-22$ per study [16] and AbbVie Data on File). Individual $C_{\max }$ and AUC values from the three weight groups of pediatric patients overlapped with those from adult patients, in whom DAAs have been shown to be safe and efficacious (S5).

\section{Acceptability}

Parental responses to the acceptability questionnaire were available for 25 (96\%) children at 
Week 12: $80 \%$ of parents required $<15 \mathrm{~min}$ to prepare and administer the study treatment; approximately half of the parents (52\%) successfully administered the study treatment with only 1-2 teaspoons of soft food; $76 \%$ of parents documented that their child had no difficulty swallowing the study treatment; and 60\% experienced no patient resistance when administering it.

\section{DISCUSSION}

$\mathrm{HCV}$ infection in children is characterized by higher rates of spontaneous viral clearance than seen in adults. In those children who develop chronic HCV infection, the progression of liver disease tends to be slow and mild and is often accompanied by elevated levels of aminotransferases, although this is not generally an indication of disease severity. However, the risk of fibrosis increases with age and with factors including obesity and HBV or HIV coinfection [1]. As a result of their availability, efficacy, and tolerability, IFN-free DAAs are now recommended to treat adolescents (aged $\geq 12$ years or weighing $\geq 45 \mathrm{~kg}$ ) with chronic HCV infection, as well as for children aged $>3$ years with chronic HCV infection [3, 7, 8, 10, 11].

To date, three clinical trials have reported the use of IFN-free DAAs in children aged $<12$ years. Two Phase 2 studies evaluated the IFN-free fixed-dose combination of ledipasvir/sofosbuvir \pm RBV in children with or without prior IFN use $[10,17]$. One study involved 92 children aged 6-11 years with chronic HCV GT1, GT3, or GT4 infection, with or without cirrhosis [17]. The other study involved 34 children aged 3-5 years with chronic HCV GT1 or GT4 infection, without cirrhosis or with unknown cirrhosis status [10]. In the 6- to 11-year age group, the overall SVR12 rate was $99 \%(91 / 92 ; 95 \%$ CI 94-100). The single child who failed to achieve SVR12 had a virologic relapse at post-treatment Week 4 . In the 3- to 5-year age group, $97 \%$ of children achieved SVR12 (33/34; 95\% CI 85-100); the only child who did not achieve SVR12 discontinued treatment after 5 days because of 'abnormal drug taste'. In both studies [10, 17], ledipasvir/sofosbuvir demonstrated a good tolerability profile, consistent with that in adolescents. Another Phase 2 study evaluated sofosbuvir and ribavirin in 54 children aged 3-11 years with chronic HCV GT2 or GT3 infection, with or without cirrhosis [11]. The majority of patients were HCV treatment-naïve $(53 / 54)$. Overall, $98 \%$ of children $(53 / 54 ; 95 \%$ CI 90-100) achieved SVR12. The one child who did not achieve SVR12 discontinued treatment after 3 days because of 'abnormal drug taste'. In this study [11], sofosbuvir and ribavirin therapy was well tolerated, and the efficacy results were comparable to those observed in adolescents and treatment-naïve adults.

The current study was conducted to assess the pharmacokinetics, safety, and efficacy of OBV, PTV, ritonavir, and DSV plus RBV for 12 weeks in children aged 3-11 years infected with HCV GT1. The results suggest that minitablet formulations of the study drugs are suitable and efficacious for chronic HCV GT1a infection in pediatric patients. Furthermore, short-term growth and development were unaffected during the period of assessment.

The weight-based pediatric doses of the DAA and ritonavir mini-tablets in children aged 3-11 years and adolescents provided generally comparable exposures across the different weight groups. The exposures in pediatric patients across the weight groups were also comparable to those in adults and adolescents [15].

The new regimen reported in this study was straightforward to prepare and administer for the majority of parents, and few children had difficulty swallowing the study treatment. There were a few limitations to the study. There were only a small number of patients enrolled and patients with cirrhosis were not included in the study population. However, the sample size and cirrhosis status of enrolled patients is similar to other studies evaluating pediatric formulations $[10,11]$. Other limitations include that the study was open-label, non-randomized, and not performed at multiple global sites. However, the current study, as well as others reporting the use of IFN-free treatment in children aged 3-11 years with HCV infection, provide valuable information on the efficacy and safety of 
several regimens, and support recommendations to use IFN-free treatments in children of this age group.

\section{CONCLUSIONS}

The mini-tablet formulations of OBV, PTV, ritonavir, and DSV plus RBV oral solution demonstrated high SVR12 rates (96.2\%) without virologic failure in children aged 3-11 years infected with HCV GT1a and without cirrhosis. This current study adds to the evidence supporting the efficacy, safety, and tolerability of pediatric-friendly formulations of DAAs in children as young as 3 years of age, and provides hope of achieving cure and prevention of progression of HCV-related liver disease in children.

\section{ACKNOWLEDGEMENTS}

Paritaprevir was identified by AbbVie and Enanta. The authors would like to express their gratitude to the patients who participated in this study and their families. The authors would like to thank the research coordinators who made this study possible, Dr. Regino P Gonzalez-Peralta for his role as an investigator on the ZIRCON study, and Linda Fredrick, of AbbVie Inc., for statistical support.

Funding. This work and the Rapid Service and Open Access Fees were supported by AbbVie. AbbVie funded the study, contributed to its design, data collection, analysis, and interpretation of the data, and participated in the writing, review, and approval of the manuscript. All authors had access to relevant data.

Medical Writing and/or Editorial Assistance. Medical writing support was provided by Paul MacCallum, PhD, of Fishawack Communications Ltd.; funded by AbbVie.

Authorship. All named authors meet the International Committee of Medical Journal Editors (ICMJE) criteria for authorship for this article, take responsibility for the integrity of the work as a whole, and have given their approval for this version to be published.

Author Contributions. PR, JZ, LL, DHL, BBY, and JPM contributed conception and design of the study; PR, LL, MMJ, DHL, SJL, MRN, JZ, and BBY contributed to data analysis; PR, LL, EKH, CDJ, MMJ, DHL, SJL, JW, MRN, and JPM were study investigators; PR, JPM, LL, BBY, and JZ contributed to the writing of the manuscript. All authors contributed to manuscript revision and read and approved the submitted version.

Disclosures. Philip Rosenthal: Grant/research support from Gilead, AbbVie, BMS, Merck, and Roche; speaker for Retrophin; consultant for Gilead, AbbVie, Intercept, Alexion, Retrophin, Albireo, Mirum, Dicerna, and Audentes. Michael R. Narkewicz: Grant/research support from AbbVie, Cystic Fibrosis Foundation, and Gilead; consultant for Vertex; stock ownership (spouse owns AbbVie stock $[<\$ 5000])$. Christopher Jolley: Institute receives research funding from AbbVie. Steven J. Lobritto: Grant/research support from AbbVie; consultant for Kadmon. Jessica Wen: Grant/research support from AbbVie, BMS, and Gilead; consultant for Gilead (DSMB). Jean P. Molleston: AbbVie, Gilead, and Shire. Evelyn K. Hsu: Grant/research support from AbbVie. Maureen M. Jonas: Consultant and grant support from Gilead; grant support from BMS, Echosens, AbbVie, Merck, and Roche. Daniel H. Leung: Grant/research support from Gilead, AbbVie, BMS, Roche, and the Cystic Fibrosis Foundation; consultant for Merck. Betty B. Yao, Jiuhong Zha, Li Liu: Employees (or former employees) of AbbVie and may hold stock or options.

Compliance with Ethics Guidelines. The study protocol and amendments were approved by the independent ethics committee or institutional review board for each study site. IRB/ IEC approval was given to the study protocol by: Columbia University Medical Center; Boston Children's Hospital; the Committees for Protection of Human Subjects at the Children's Hospital of Philadelphia; the Western Institutional Review Board (WIRB) at the University of 
Florida; WIRB at Seattle Children's Hospital; WIRB at Baylor College of Medicine; WIRB at the Children's Hospital Colorado; the Indiana University Office of Research Administration, Human Subjects Office; the Human Research Protection Program IRB at the University of California, San Francisco. The study was designed and conducted in accordance with the International Conference on Harmonization Good Clinical Practice guidelines and the ethical principles of the Declaration of Helsinki. Parents or legal guardians provided written informed consent, and participants gave assent, as appropriate for their age and country. All authors had access to study data and reviewed and approved the final manuscript.

Data Availability. AbbVie is committed to responsible data sharing regarding the clinical trials we sponsor. This includes access to anonymized, individual and trial-level data (analysis data sets), as well as other information (e.g., protocols and Clinical Study Reports), as long as the trials are not part of an ongoing or planned regulatory submission. This includes requests for clinical trial data for unlicensed products and indications. This clinical trial data can be requested by any qualified researchers who engage in rigorous, independent scientific research, and will be provided following review and approval of a research proposal and Statistical Analysis Plan (SAP) and execution of a Data Sharing Agreement (DSA). Data requests can be submitted at any time and the data will be accessible for 12 months, with possible extensions considered. For more information on the process, or to submit a request, visit the following link: https://www.abbvie.com/ourscience/clinical-trials/clinical-trials-data-and-in formation-sharing/data-and-information-shar ing-with-qualified-researchers.html.

Open Access. This article is licensed under a Creative Commons Attribution-NonCommercial 4.0 International License, which permits any non-commercial use, sharing, adaptation, distribution and reproduction in any medium or format, as long as you give appropriate credit to the original author(s) and the source, provide a link to the Creative Commons licence, and indicate if changes were made. The images or other third party material in this article are included in the article's Creative Commons licence, unless indicated otherwise in a credit line to the material. If material is not included in the article's Creative Commons licence and your intended use is not permitted by statutory regulation or exceeds the permitted use, you will need to obtain permission directly from the copyright holder. To view a copy of this licence, visit http://creativecommons.org/licenses/by$\mathrm{nc} / 4.0 /$.

\section{REFERENCES}

1. Squires JE, Balistreri WF. Hepatitis $C$ virus infection in children and adolescents. Hepatol Commun. 2017;1(2):87-98.

2. Benova L, Mohamoud YA, Calvert C, Abu-Raddad LJ. Vertical transmission of hepatitis C virus: systematic review and meta-analysis. Clin Infect Dis. 2014;59(6):765-73.

3. Indolfi G, Hierro L, Dezsofi A, Jahnel J, Debray D, Hadzic N, et al. Treatment of chronic hepatitis C virus infection in children: a position paper by the Hepatology Committee of European Society of Paediatric Gastroenterology, Hepatology and Nutrition. J Pediatr Gastroenterol Nutr. 2018;66(3): 505-15.

4. Khaderi S, Shepherd R, Goss JA, Leung DH. Hepatitis $C$ in the pediatric population: transmission, natural history, treatment and liver transplantation. World J Gastroenterol. 2014;20(32): 11281-6.

5. Gower E, Estes C, Blach S, Razavi-Shearer K, Razavi H. Global epidemiology and genotype distribution of the hepatitis $\mathrm{C}$ virus infection. J Hepatol. 2014;61(1 Suppl):S45-57.

6. Delgado-Borrego A, Smith L, Jonas MM, Hall CA, Negre B, Jordan SH, et al. Expected and actual case ascertainment and treatment rates for children infected with hepatitis C in Florida and the United States: epidemiologic evidence from statewide and nationwide surveys. J Pediatr. 2012;161(5):915-21.

7. AASLD-IDSA. Recommendations for testing, managing, and treating hepatitis C. https://www. hcvguidelines.org. Accessed Nov 21, 2019.

8. European Association for the Study of the Liver. EASL recommendations on treatment of hepatitis C 2018. J Hepatol. 2018;69(2):461-511. 
9. Batchelor HK, Marriott JF. Formulations for children: problems and solutions. Br J Clin Pharmacol. 2015;79(3):405-18.

10. Schwarz KB, Rosenthal P, Murray KF, Honegger JR, Hardikar W, Hague R, et al. Ledipasvir-sofosbuvir for 12 weeks in children 3 to $<6$ years old with chronic hepatitis C. Hepatology (Baltimore, MD). 2020;71(2):422-30.

11. Rosenthal P, Schwarz KB, Gonzalez-Peralta RP, Lin $\mathrm{CH}$, Kelly DA, Nightingale S, et al. Sofosbuvir and ribavirin therapy for children aged 3 to $<12$ years with hepatitis $C$ virus genotype 2 or 3 infection. Hepatology (Baltimore, MD). 2020;71(1):31-43.

12. Gilead Sciences Inc. HARVONI (ledipasvir and sofosbuvir) US prescribing information. Available from: https://www.accessdata.fda.gov/drugsatfda docs/label/2019/205834s031,212477s001lbl.pdf.

13. Gilead Sciences Inc. SOVALDI (sofosbuvir) US prescribing information. Available from: https://www. accessdata.fda.gov/drugsatfda_docs/label/2019/204 671s016,212480s001lbl.pdf.
14. AbbVie Inc. TECHNIVIE (ombitasvir, paritaprevir, and ritonavir) US prescribing information. https:// www.accessdata.fda.gov/drugsatfda_docs/label/ 2018/207931s012lbl.pdf.

15. Leung DH, Wirth S, Yao BB, Viani RM, GonzalezPeralta RP, Jonas MM, et al. Ombitasvir/paritaprevir/ritonavir with or without dasabuvir and with or without ribavirin for adolescents with HCV genotype 1 or 4 . Hepatol Commun. 2018;2(11):1311-9.

16. Lalezari J, Sullivan JG, Varunok P, Galen E, Kowdley $\mathrm{KV}$, Rustgi $\mathrm{V}$, et al. Ombitasvir/paritaprevir/ $\mathrm{r}$ and dasabuvir plus ribavirin in HCV genotype 1-infected patients on methadone or buprenorphine. J Hepatol. 2015;63(2):364-9.

17. Murray KF, Balistreri WF, Bansal S, Whitworth S, Evans HM, Gonzalez-Peralta RP, et al. Safety and efficacy of ledipasvir-sofosbuvir with or without ribavirin for chronic hepatitis $\mathrm{C}$ in children ages 6-11. Hepatology (Baltimore, MD). 2018;68(6): 2158-66. 